\title{
Enantioconvergent Photoinduced Copper-Catalyzed Synthesis of Chiral Secondary Amides
}

\section{Key words}

copper catalysis

amidation

enantioconvergence

visible light
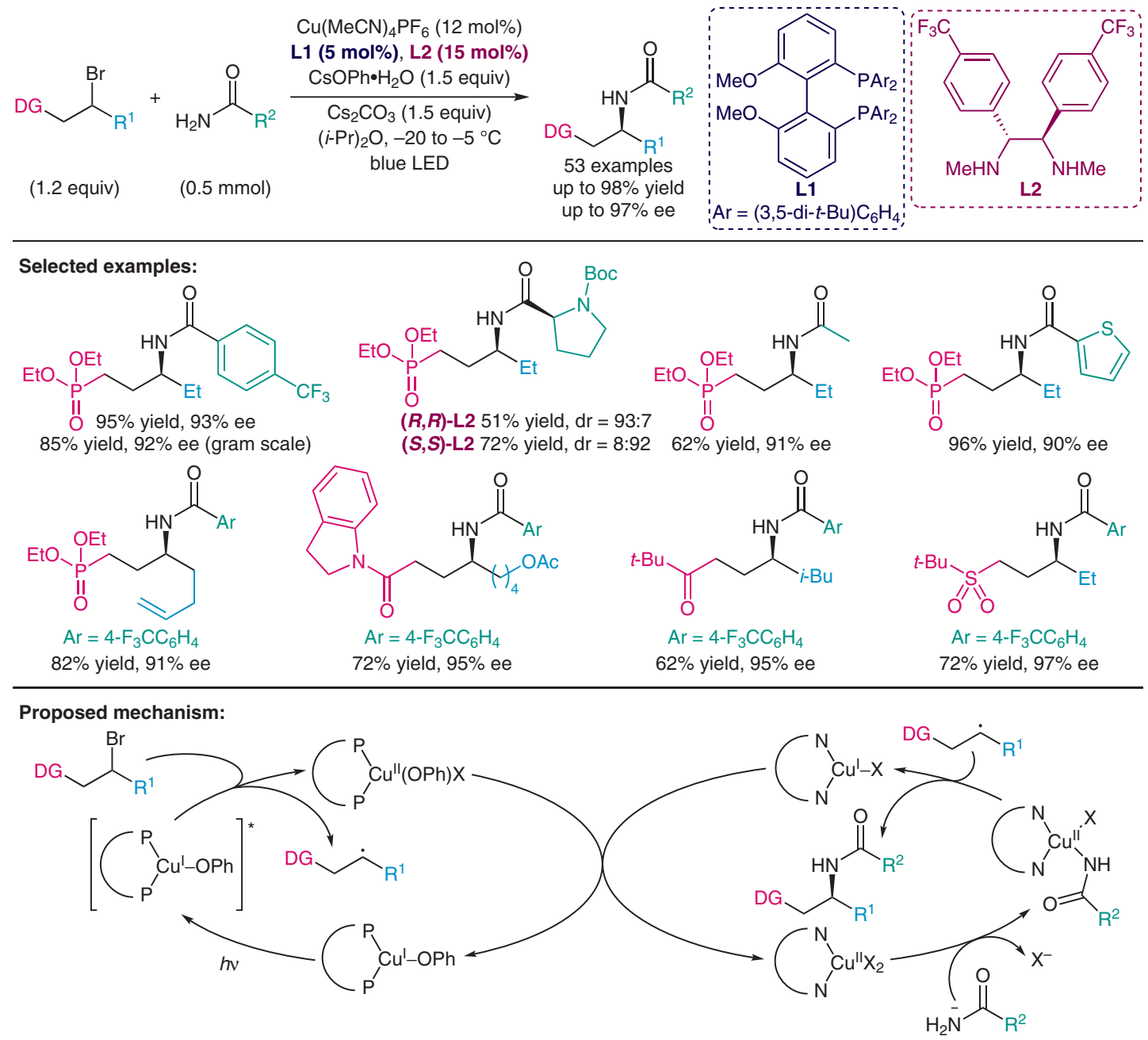

Significance: Under blue-LED irradiation, the enantioconvergent $\mathrm{C}-\mathrm{N}$ coupling of racemic alkyl electrophiles with primary amides using copper catalysis has been described. A variety of chiral secondary amides were formed, with a Lewis-basic functional group on the electrophile component to allow for differentiation of similar alkyl substituents. Through control studies, the stereochemistry of the amide product was shown to be determined by the diamine ligand, irrespective of the stereochemistry of the bisphosphine ligand or electrophile.
Comment: Extensive mechanistic studies were conducted to elucidate the role of the three ligands used in the catalyst system. Together, the bisphosphine and phenoxide ligands form the photocatalyst responsible for activating the $\mathrm{C}-\mathrm{Br}$ bond, whereas the diamine ligand was found to be necessary for forming the $\mathrm{C}-\mathrm{N}$ bond and controlling stereochemistry. The involvement of a radical intermediate was supported by trapping and cyclization experiments. 